\title{
La Política Cultural Cubana (y su Cuestionamiento) en la Narrativa Cubana Contemporánea: La grieta (2018), Las palabras perdidas (1992), Las voces y los ecos (2004) y La noria (2013).
}

The Cuban Cultural Policy

(And its Questioning) in Contemporary

Cuban Narrative: La grieta (2018),

Las palabras perdidas (1992), Las voces

y los ecos (2004) y La noria (2013).

\section{Delia García Muñoz}

Florida International University

\section{Resumen}

En las novelas que nos ocupan -La grieta, Las palabras perdidas, Las voces y los ecos y La noria-, los personajes tratan de llevar a cabo sus propósitos artísticos y profesionales bajo la presión de un aparato estatal que los sanciona o ilegitima. La problemática relación entre el Estado cubano y la creación artística a partir del Quinquenio Gris permite, a través de la ficción, un cuestionamiento de la política cultural cubana iniciada en los sesenta y una reflexión sobre el papel de la intelectualidad en la isla. Dicha conflictividad encuentra su etapa más cruenta y represiva en los llamados años del Quinquenio Gris (1971-1976). Persecución que dejó mella en la sociedad del momento, pero también en las generaciones posteriores de escritores, que la retomarán como motivo en sus novelas desde diversas perspectivas.

Palabras clave: Política Cultural, Narrativa Contemporánea, Novelas, Cuba.

\begin{abstract}
In the novels that concern us - La grieta, Las palabras perdidas, Las voces y los ecos y La noria-, the characters try to carry out their artistic and professional purposes under the pressure of a State Apparatus that sanctions or makes them illegitimate. The problematic relationship between the Cuban State and artistic creation since the Quinquenio Gris allows, through fiction, a questioning of Cuban Cultural Politics that began in the sixties, and a reflection on the role of the intellectuals on the island. The conflictive relationship between the artist and the Cuban State finds its most repressive stage in the so-called years of the Quinquenio Gris (1971-1976). This persecution of artists left a dent in the society of the moment but also in later generations of writers, who will take it up again in their novels from different perspectives.
\end{abstract}

Key words: Cultural Policy, Contemporary Narrative, Novels, Cuba.

La conflictiva relación entre el artista y el Estado cubano encuentra su etapa más cruenta y represiva en los llamados años del Quinquenio Gris (1971-1976). Dicha persecución de artistas dejó mella en la sociedad del momento, pero también en generaciones posteriores de escritores, que lo retomarán en sus novelas desde diversas perspectivas. En las novelas consideradas por el presente artículo $-\mathrm{La}$ grieta, Las palabras perdidas, Las voces y los ecos y La noria-, los personajes tratan de llevar a cabo sus propósitos artísticos y profesionales bajo la presión de un aparato estatal que los sanciona o ilegitima. Las dificultades suscitadas a partir del Quinquenio Gris permiten, a través de la ficción, un cuestionamiento de la política cultural cubana iniciada en los sesenta y una reflexión sobre el papel de la intelectualidad en la isla.

En este orden, el triunfo de la Revolución en enero de 1959 y la alineación de Cuba con el bloque socialista en los años posteriores tuvieron un impacto decisivo en la cultura cubana que causó profundas transformaciones. El ascendente nacionalismo trajo consigo un proceso de "descolonización" de los elementos extranjeros (provenientes de Europa y de los Estados Unidos), al tiempo que supuso la búsqueda y construcción de la incipiente identidad cubana revolucionaria.

Para ello, el Estado adoptó numerosas medidas y creó instituciones que salvaguardaran la cultura nacional, a saber: el Instituto Cubano del Arte e Industria Cinematográficos (ICAIC), la Unión de Escritores y Artistas de Cuba (UNEAC), el Instituto Superior de Arte (ISA), el Ballet Nacional de Cuba, entre otros. Con el paso del tiempo, no se hicieron esperar los interrogantes sobre la función de los intelectuales dentro de la Revolución y en el año 1961 tuvo lugar el conocido discurso de Fidel Castro, Palabras a los intelectuales, que sentó la primera piedra 
de la que sería la política cultural cubana de la Revolución, y cuyo lema fuera: "dentro de la Revolución todo, fuera de la Revolución nada”. Más tarde, en el primer Congreso Nacional de Educación y Cultura de 1971 se establecería la nueva política del gobierno que regularía el arte y la literatura. El aparato ideológico y estético se adoptó de la Unión Soviética y la cultura empezó a considerarse un arma al servicio del discurso oficial.

Si bien, durante los años 70 se promulgó el realismo socialista soviético, corriente estética que la intelectualidad soviética adoptó con el fin de ocultar la historia anterior y transformar la realidad. No obstante, como en la isla antillana las circunstancias históricas habían sido muy distintas a las de la URSS, no todos los intelectuales compartieron dicha tendencia. En este sentido, Rafael Rojas (2009, p. 77) explica la sovietización de las ciencias sociales y sostiene que: "la ideología marxista-leninista y, en especial, su tratamiento académico en la Universidad de Lomonosov y otras instituciones de las ciencias sociales en Moscú, era el área donde la potencia soviética practicaba su hegemonía sobre Cuba”.

Por su parte, para Albert Otto Hirsman, (como se citó en De Ferrari 2017, p.18) la lealtad del pueblo comunista se basa tanto en la suspensión del derecho a la voz, esto es, la pérdida de la libertad de expresión, como en el derecho de salida, es decir, el derecho a abandonar el país. Como propone Guillermina De Ferrari (2017, p. 20), es posible aplicar esta índole de "contrato social" a la relación del Estado cubano con los intelectuales y artistas:

Se deduce en este marco que existe un contrato social socialista específico entre el estado y el artista, según el cual el estado facilita formas de subsistencia, de producción artísticas y medios/espacios de difusión -en forma más generosa que el capitalismo-, a cambio de la lealtad de un artista con derecho parcial de voz y de salida. El estado se reserva además el derecho de silenciar al artista, lo que equivale a neutralizarlo o eliminarlo como figura pública. En resumen, el estado no confía en el artista.

Por lo tanto, la intelectualidad, entendida como oficio abstracto, no tenía cabida en la sociedad revolucionaria si no asumía una actitud comprometida con la ideología de la Revolución. Esta desconfianza del estado es una característica común en las novelas que se examinan. La eliminación del autor como figura pública nos remite a los personajes "vaporizados" de Orwell en su libro 1984.
Seymour Menton (1975, p. 139) analiza la narrativa cubana de la Revolución y concluye que los temas más frecuentes después de 1959, entre otros, son: "reluctance of the burgeoisie to accept revolutionary changes", tanto como la "counter insurgency campaign of the early 196o". Este autor expone, por una parte, que los escritores de este periodo se preocupan por el retrato de la Cuba prerrevolucionaria, por lo que el movimiento urbano clandestino contra Batista recibe más atención que la guerrilla de Fidel Castro en Sierra Maestra. Lo característico de estas novelas era que en ellas se evitan los temas controversiales de la Revolución. Por otro lado, señala que los trabajos publicados en Cuba son ambiguos mientras que los divulgados en el extranjero son "diatribas anticomunistas", entre las que se encuentran algún intento serio de retratar las condiciones en Cuba. Sobre la censura y persecución de escritores en los setenta, han sido estudiados por extenso los casos del poeta Heberto Padilla $^{1}$, del dramaturgo Antón Arrufat $^{2}$, de los escritores Reinaldo Arenas ${ }^{3}$ y Cabrera Infante, así como del documental P.M. (Pasado Meridano) ${ }^{4}$ del free cinema cubano, entre otros muchos ejemplos de persecución y "parametración”.

El comienzo de la novela La grieta de Reinaldo Escobar (2018, p. 18) narra la división que se formó en una clase de periodismo por el debate de la novela $E l$ Gran Burundún Burundá ha muerto de Jorge Zalamea. El debate polémico surgió por la interpretación de la trama de la obra: "el gobierno de un dictador que había llegado al colmo represivo de prohibir, mediante decreto oficial, el uso de la palabra articulada”. Por un lado, un grupo encontraba entre líneas una recriminación a los regímenes totalitarios. Por el otro lado, el resto denunciaba que el texto hacía una crítica "excesivamente generalizada al concepto de dictadura", lo que suponía "una desviación ideológica de la línea oficial”. Ya que, aunque no se mencionara, se podía interpretar como una crítica a la dictadura del proletariado, "vía inexcusable para tomar y mantener un poder capaz de alcanzar los elevados objetivos de la Revolución Socialista". En este fragmento observamos la incorporación del análisis ideológico en la interpretación de un texto literario hecha por los estudiantes y los posicionamientos que esta provoca.

Finalmente, "la polémica llegó a los extremos en que los más duros de una y otra posición se hicieron acusaciones personales de librepensadores unos y de oficialistas otros" (Escobar, 2018, p. 18). Los dos grupos en los que se divide la clase-"los oficialistas" 
y "los librepensadores" - nos sirven para ilustrar la división de la intelectualidad cubana que se originó en los setenta como consecuencia de la política cultural. Por lo que resulta relevante traer al caso la definición de estos grupos que distingue el narrador de La grieta:

Los oficialistas eran definidos por sí mismos, el vivo retrato de la lealtad política. Se sentían capaces de dedicar su vida a la Revolución, entendiendo que era válido pasar por encima de cualquier consideración momentánea, si hacerlo acercaba de alguna forma el futuro luminoso por el que luchaban. Se creían perdonados de antemano por la historia, ante cualquier error que pudieran cometer durante la ejecución de la gran tarea, porque a fin de cuentas, solo quienes se entregan de lleno están en la posibilidad de cometer errores. No podían admitir la existencia de ninguna duda, no demandaban una prueba de lo que se anunciara como logrado, ni una garantía de lo que se prometiera como seguro, porque la fe en quienes dirigían el proceso significaba la espina dorsal de su disciplina militante. Por mantener la pureza de esa fe estaban dispuestos a cualquier cosa a cambio de nada. Su vida era un sacerdocio con votos perpetuos de austeridad, obediencia y absoluto desprendimiento. Hacer realidad su causaun ideal perfecto e indiscutible, válido para ellos y para los demás-constituía el motivo esencial de sus vidas, y no porque les conviniera, sino porque lo consideraban históricamente irrevocable (Escobar, 2018, pp.18-9).

Este grupo representa la fe del contrato social socialista que expone Guillermina De Ferrari (2017, p. 19), según el cual los ciudadanos, a cambio de seguridad política y económica, han renunciado "a sus garantías a priori" y deben "confiar en que el gobierno cumplirá con su parte del trato después”. En el caso cubano, esta fe se depositó en la utopía de forjar un nuevo país que la Revolución garantizaba y en los valores del hombre nuevo, tales como el sacrificio, la austeridad o el amor por el trabajo. Al comparar la vida de este grupo con "un sacerdocio" el narrador evoca el cariz religioso con el que se evoca, en ocasiones, la Revolución en el imaginario popular. Del otro lado, los librepensadores se definen de esta manera:

Los llamados librepensadores, al igual que los oficialistas, tenían un magnífico concepto de sí mismos. Se sentían los defensores del más sagrado derecho que puede tener un hombre: el derecho a expresar sus ideas, hijo de otro derecho no menos sagrado: el de someter a examen del pensamiento todo acontecer pasado, presente o por venir; los principios morales, las categorías científicas, las leyes, las creencias y los dogmas. Esta libertad en la forma de pensar y de expresar lo pensado, constituía una conquista extensible a ciertas libertades en la conducta. A ellos les tocaba defender las libertades ya alcanzadas y propugnar el establecimiento de aquellas no logradas aún, principalmente las individuales. Preconizaban la necesidad de la cultura artística como un resorte para despertar en el hombre el tesoro de sus valores humanos (Escobar, 2018, p. 19).

En la novela, a través de la autoficción, Reinaldo Escobar acaba demostrando que en la política cultural no habrá cabida para los librepensadores. Este destino, nos permite establecer una analogía: por una parte, los librepensadores de la novela y, de otra, la intelectualidad que fue asociada con la burguesía y criminalizada por su pensamiento crítico, en la medida en que se les consideraba como enemigos de la Revolución y aliados del imperialismo. Un personaje representativo de esta idea de burguesía lo encontramos en el protagonista de Memorias del Subdesarrollo, de Edmundo Desnoes. En el libro La grieta, el narrador enumera los juicios negativos que cada grupo extrae del contrario. Los oficialistas juzgan "las posiciones pluralizantes" de los librepensadores como "una malsana y claudicante intención de socavar la indispensable unidad del pueblo, unánimemente enfrascado en el propósito de alcanzar los elevados objetivos de la Revolución Socialista" y "las pretensiones de elevación espiritual podrían catalogarse de burda autosuficiencia; la sobrevaloración de la cultura artística, un truco intelectualoide para descalificar la cultura política”. Hemos de destacar que es una lectura literaria la que conduce a los estudiantes a la necesidad de posicionarse ideológicamente en dos grupos enfrentados. De forma que, este pasaje refleja la exclusión de los artistas o escritores que consideran el arte como un fin en sí mismo, ya que esto supone una "pretensión de elevación espiritual" que se aleja de los "objetivos de la Revolución Socialista" y pone en peligro "la cultura política”. Mientras que, entre otras recriminaciones, los librepensadores consideran que:

el afán por la pureza no era más que puro sectarismo; la proclamada fe en el futuro, un pasaporte falso para gozar en el presente; la intransigencia, un miedo incontenible a ser desenmascarados; el desprendimiento, un precio-avariciosamente calculado- para obtener el mérito como puente a las ventajas y privilegios" (Escobar, 2018, p. 19). 
De momento, se han examinado dos posturas enfrentadas: oficialistas y librepensadores. Ahora bien, ¿qué sucede con las posiciones intermedias?

El protagonista de La grieta da cuenta de lo excluyente que es esta realidad polarizada. En parte, admira la entrega con la que los oficialistas "se dedicaban a la causa" y "su voluntad inquebrantable", al tiempo que, comparte con los librepensadores "el amor por la libertad de pensamiento, palabra y acción; la tolerancia hacia las posiciones ajenas y la convicción de que cualquier proyecto puede ser infinitamente mejorado si se expone a la luz de las diversas opiniones" (Escobar, 2018, p. 21). Al intentar exponer su opinión en el debate, Antonio Martínez "sintió que bajo sus pies se perfilaba y ensanchaba una grieta entre dos rocas monolíticas e irreconciliables, cuyo único propósito aparente eran el de diferenciarse la una de la otra”. Desde el primer capítulo, advertimos que le será imposible sostenerse, como pretendía, con un pie en cada borde.

En la novela, Antonio Martínez no podrá terminar la carrera de periodismo con su promoción porque, a pesar de mostrar su compromiso con la revoluciónparticipa en las labores agrícolas e industriales, se alista voluntariamente al ejército, colabora en la Alfabetización-, es tachado de hipercrítico por sus ideas relativas a la libertad de prensa. Aunque consigue superar los juicios ideológicos y ejercer como periodista, tras numerosos incidentes es inhabilitado de su profesión. Durante la narración, el autor implícito contextualiza la acción en el marco del Quinquenio Gris:

Tres meses después de aquella catarsis comenzó el proceso. Por esos días la Seguridad del Estado detuvo al poeta Heberto Padilla que dos años antes había protagonizado un escándalo, al ser premiado en un concurso nacional de literatura su libro Fuera del juego. Ahora se le acusaba, de haber sacado del país, en forma ilegal y subrepticia, una novela políticamente conflictiva para publicarla en el extranjero. Desde lo más alto de La Máxima instancia se dio una señal de parar en seco "los excesos de criticismo" en que había terminado aquel "saludable proceso de autocrítica" iniciado tras el fracaso de La Gran Zafra (Escobar, 2018, p. 41).

Fuera del juego de Heberto Padilla fue el poemario premiado por la Casa de las Américas en 1968. Fue publicado con un prefacio que mostraba el desacuerdo de la UNEAC por "posiciones ambiguas en problemas fundamentales que conciernen a la Revolución
Cubana” (Reed, 1991, p. 99). El mismo Reed divide el caso Padilla en tres fases. Según el investigador, el poeta es encarcelado en 1971 por algunos poemas del recital del poemario Provocaciones y por estar trabajando en una novela crítica que incluía a Fidel Castro como personaje alegórico (Reed, 1991, p. 103). Por lo tanto, podemos situar el fragmento en su tercera etapa. El "caso Padilla" se ha vuelto uno de los símbolos en la narrativa cubana reciente que ilustra la represión y la condena al olvido que sufrieron muchos artistas en la isla al haber sido juzgados por el contenido de sus obras. Este tipo de control mental que los artistas debían desarrollar en contextos totalitarios para no ser censurados o castigados, como explica Zygmunt Bauman (2019), se escenifica en la aludida 1984 con la técnica del doble-pensar.

En lo que respecta a la protagonista de Las voces y los ecos de Aida Bahr (2006), es esta una joven que comienza la carrera de letras en la Universidad de Oriente. En su primer año de carrera, le niegan una ayudantía por "problemas de autosuficiencia" (Bahr, 2006, p. 40). Quiere convertirse en escritora y forma parte de una tertulia y de una compañía de teatro, para la que decide escribir una obra. La obra es una comedia ligera basada en sus vivencias en la beca, "un retrato más o menos amable de la vida en las becas" (Bahr, 2006, p. 96). El conflicto del drama se produce por un episodio que inventa que provoca un enfrentamiento con una profesora. La obra es muy bien acogida por sus compañeros de la compañía y la animan a que la presente a un concurso en Panamá. Sin embargo, cuando en la universidad tienen noticia de este hecho, le recuerdan que la obra tiene que pasar una revisión de su departamento primero.

La Dra. Cáceres, con la que la protagonista trabaja, intenta disuadirla de que la envíe al extranjero alegando que: "si no fuera por este acoso continuo del imperialismo, no tendríamos que ser tan cuidadosos. Yo no creo que tu obra tenga nada de malo, incluso es optimista, pero hay que evitar darle armas al enemigo" (Bahr, 2006, p. 104). La profesora cree que la visión de las aulas cubanas en el exterior podría verse tergiversada. Siguiendo su advertencia, decide no participar en el concurso, pero para entonces ya ha despertado sospechas en la asociación de estudiantes y varias compañeras la denuncian ante el decano y su puesto en la Universidad peligra. En su defensa sobre la obra en la asamblea, arguye: "no se habló de problemas ideológicos. Me dijeron que la obra podía ser tergiversada y no quise que pasara eso. No quise que se utilizara una obra mía para atacar a la revolución. Pero hasta Martí ha sido 
tergiversado" (Bahr, 2006, p. 165). En esta ocasión, la protagonista añade una crítica a la reinterpretación de los símbolos nacionales como José Martí. Al intentar defenderse, siente "ser sometida a la Inquisición y declarada hereje" (Bahr, 2006, p. 121). El proceso al que es sometida la protagonista se compara con los castigos de la Inquisición y equiparan las directrices revolucionarias a los dogmas religiosos. Esta comparación ya la notamos en la definición que ofrecía el narrador de La grieta sobre los "oficialistas".

Con motivo de estos acontecimientos que la angustian, la protagonista reflexiona sobre la realidad del pecado original: "hay pecados originales con los que se nace, otros se adquieren en la vida, a menos que uno sea tan consciente de ellos, y les tenga tanto miedo, que trate a toda costa de evitarlos" (Bahr, 2006, p. 124). Aunque no haga explícitamente una mención al discurso del Che Guevara, la idea nos remite al pecado original que el Che atribuye a los intelectuales cubanos que han nacido antes de la Revolución.

Su reflexión concluye en ese sentido: "yo había creído que ser intelectual era algo muy importante y respetado, ¿no eran intelectuales Carlos Marx y el propio Fidel? Me faltaban por descubrir algunas cosas" (Bahr, 2006, p. 125). Su pregunta refleja la función incierta del intelectual en los setenta, sobre la que la política cultural ya había comenzado a establecer sus directrices en los sesenta. Cabe preguntarnos, ¿por qué si el personaje es fiel a la causa revolucionaria es expulsada de la Universidad por ser "autosuficiente" y por una interpretación malintencionada de su obra? Esta pregunta retórica pone de manifiesto una de las fallas de la política cultural de los setenta: la ambivalencia de participar en la Revolución y sin embargo, ser castigado por diferencias estéticas entendidas como ideológicas.

Justamente, el personaje de El Flaco de Las palabras perdidas de Jesús Díaz (1996) utiliza el argumento del pecado original del Che para convencer al director de un periódico de que le permita dirigir un suplemento cultural con otros profesores jóvenes de la Universidad:

Venía, dijo en un tono más alto del que hubiese deseado, a dar cuenta de un hecho, de un acontecimiento. Una nueva generación de escritores había surgido con la revolución, un grupo en el que, por primera vez desde el año cincuenta y nueve, las vanguardias política y artística se fundían en un todo indisoluble. Le hablaba de jóvenes que no tenían compromisos con el pasado y que podían decir la verdad porque no tenían nada que perder. El compañero director conocía seguramente la tesis del Che: el pecado original de los intelectuales cubanos era el no ser auténticamente revolucionarios. Pues bien, ellos, los jóvenes hijos de la revolución, sí lo eran. Y por tanto, eran también los únicos capaces de responder a la demanda de una crítica activa y una expresión militante de la contemporaneidad en el arte y la literatura (Díaz, 1996, p. 70).

Con su argumentación, consigue que el director acepte y durante toda la novela el grupo de amigos junto con Una, una nueva integrante, trabaja en la revista cultural "El Güije", que busca aunar literatura cubana de alta calidad. Esta generación se considera heredera de Lezama Lima y quieren "matar al padre artístico" simbólicamente. Como hijos de la revolución, no tenían deudas con la dictadura de Batista y querían desarrollar nuevas formas literarias que aportaran una gran calidad estética y estilística a las letras cubanas. Cada uno de los güijes tiene un criterio personal y una idea sobre el panorama literario nacional, Andrea Mellado (2007, p. 73) sintetiza estos gustos:

El Rojo, por ejemplo, desea inventar un nuevo idioma para así poder "romper la prisión del realismo, la servidumbre de la anécdota, la miseria del color local" (19), pobrezas que asocia al conversacionalismo y coloquialismo que "banalizaban hasta el hastío la joven poesía cubana creando la desoladora impresión de que todos los poemas estaban escritos por el mismo pésimo poeta" (20). El Gordo, en cambio, defiende el coloquialismo y critica la búsqueda del Flaco, que califica de "epigonal, falsa, vacía y extranjerizante" (114). El Flaco quiere crear una novela total "que incluya todos los géneros literarios, poesía, cuento, periodismo, ensayo" (38); busca que sus textos aporten "realmente algo nuevo" (107). Una, por su parte, pretende escribir dos libros complementarios (un ensayo y un poemario) que funcionen como "homenajes personales a ciertas escritoras" (183).

Sin embargo, tristemente, todo el esfuerzo y dedicación que el grupo dedica se ve frustrado por el trasfondo político. Tras revisar el suplemento, el director no le da el visto bueno porque varios relatos y poemas atentan contra el sentido revolucionario. El Flaco intenta defender los contenidos y sentencia que el informe "era una sarta de sofismas" y que el autor criticado era un "poeta puro" que no entendía de política: "sus textos no eran sino metáforas universales, escritas para ser leídas e interpretadas de diversas maneras en distintos países y épocas" 
(Díaz, 1996, p. 336). A diferencia del personaje Antonio Martínez, el Flaco no era consciente de la grieta insalvable que separaba su concepción de la literatura, atribuible a un "librepensador" de la del director, claramente "oficialista". El fracaso de la revista nos devuelve el cuestionamiento de la política cultural de los setenta. Pero la novela va más allá, y muestra al lector las consecuencias negativas y paralizantes que la censura (tanto artística como pública) tiene en los güijes y los sitúa como víctimas de un sistema represivo. Además, ofrece un breve compendio de los autores prohibidos en los setenta, lo que supone una crítica al modelo estalinista que se había intensificado en la política cultural en aquella época.

Las novelas que hemos señalado hasta el momento abordan la compleja situación entre los intelectuales y el estado en los setenta haciendo uso de un narrador omnisciente, en el caso de La grieta y Los pasos perdidos, mientras que en Las voces y los ecos encontramos una narradora protagonista. Reinaldo Escobar (1947), Jesús Díaz (1941), Aida Bahr (1958) vivieron el periodo sobre el que escriben lo que los acerca a la dimensión testimonial. Sobre todo, en la obra de Reinaldo Escobar que contiene muchos elementos autobiográficos y su personaje Antonio Martínez confiesa en la última página estar escribiendo su vida en una novela con el alter ego "Reinaldo Escobar".

Sin embargo, el autor de la última novela que nos ocupa, Ahmel Echevarría (2013), no vivió durante el Quinquenio Gris. Su obra, La noria, constituye una recuperación de la memoria y un trabajo de archivo que usa como estrategias narrativas la metaficción y la intertextualidad, estrategias características de su generación. Mónica Simal (2017) analiza la obra en relación con el contexto histórico presente del escritor del siglo XXI que retorna al pasado para indagar sobre la condición del intelectual cubano en los últimos cuarenta años. La autora considera que la función de la obra es "hacer un homenaje al pasado y a sus protagonistas tras el "plagio" de sus voces escriturales y otras, como ocurre con la de Cortázar, y poder así elaborar una crítica político-social. Esta consciente operación de Echevarría es su conjuro contra la mala memoria oficialista, especialmente durante su acto de exhumación del quinquenio gris" (Simal, 2017, p. 69).

Simal (2017, p. 69) señala que el género que El Maestro escoge para su cuento, el género policíaco, y que el mismo Echevarría usa para su novela, supone una vuelta a los setenta. Nos encontramos ante un mise en abyme que representa la evolución de la política cultural cubana:
Ya no se trata de recrear una historia donde el policía revolucionario se encuentra luchando en contra de los rezagos burgueses presentes en la sociedad. En un movimiento opuesto, El maestro en su cuento, y en general Echevarría en su novela, recrean la persecución policial del Estado revolucionario contra el escritor, cuyo delito es, precisamente, su escritura. (Simal, 2017, p. 70).

Pareciera que Echevarría tratase de emular a Cervantes, y en lugar de recoger los géneros literarios de su época, recogiera en su novela un estudio de la evolución de la política cultural de su país a partir de los setenta. Duanel Díaz (2009, p. 152) apunta que el éxito de la novela policiaca vio su auge entre el 1971- 1983. Este éxito, continúa, no se puede entender como un éxito del mercado sino como un logro del estado.

Dada la represión sobre los temas que se alejaran de lo marcado por la política cultural, una de las pocas alternativas que quedaba que no fuera censurada era, precisamente, la novela policiaca. El género se llenó de connotaciones singulares en Cuba, de hecho, según Díaz, fue un "producto de la Iniciativa de la Dirección Política del Ministerio del Interior" que comenzó a convocar en el año 1972 un concurso anual de novela policial, "con el propósito de promover obras que tendrán un carácter didáctico y serán un estímulo a la prevención y vigilancia de todas las actividades antisociales o contra el poder del pueblo" (Díaz, 2009, p. 153). Por supuesto, en la actualidad, el género se ha visto reinterpretado y enriquecido por autores tales como el escritor Leonardo Padura.

En definitiva, hemos visto que la política cultural cubana y su cuestionamiento es un tema que ha sido revisitado a lo largo de los años por autores de diferentes generaciones. En las novelas escogidas, los personajes son castigados a causa de sus concepciones o de las ideas que pudieran despertar sus textos. Esto es, por manifestar "diversionismo ideológico" o porque sus textos pudieran ser una posible arma en manos "del enemigo" de la Revolución. Cabe preguntarse, ¿a qué respondería esta tendencia de ficcionalizar la etapa represiva de los años setenta en la narrativa cubana? En los noventa, con la llegada de la crisis del Periodo Especial, todo este sistema que vivieron los autores en los setenta queda definitivamente obsoleto y la literatura encuentra nuevas formas subversivas en la novela que demuestran la caída del hombre nuevo. Con este fin, puede consultarse el estudio de Sonia Behar (2009). Sin embargo, ¿por qué persiste esta tendencia de ficcionalizar la 
etapa represiva de los años setenta en la narrativa cubana a lo largo del tiempo? La respuesta se abre camino en la literatura del desencanto que refleja la crisis de una sociedad en transición y el papel de las letras en la recuperación de la memoria histórica de esta. Es en ese marco, donde podemos situar estas ficciones que problematizan los peligros de la instrumentalización del arte.

\section{Referencias}

Arango, A. (2008). "Con tantos palos que te dio la vida: Poesía, censura y persistencia”. La política cultural del período revolucionario: memoria y reflexión. La Habana: Centro Teórico-Cultural Criterios.

Bähr, A. (2006). Las voces y los ecos. La Habana: Ediciones Unión.

Bauman, Z. y Donskis. L. (2019). Maldad líquida. Barcelona: Editorial Paidós.

Behar, S. (2009). La caída del hombre nuevo. Narrativa cubana del Período Especial. Nueva York: Peter Lang Publishing.

De Ferrari, G. (2017) Comunidad y Cultura en la Cuba Postsoviética. Madrid: Editorial Verbum.

Desnoes, E. (2006). Memorias del subdesarrollo. Sevilla: Mono Azul.

Díaz, J. (1996). Las palabras perdidas. Barcelona: Editorial Anagrama.

Díaz-Infante, D. (2009). Palabras del trasfondo. Intelectuales, literatura e ideología en la Revolución Cubana. Editorial Colibrí.

Escobar, R. (2018). La Grieta. Madrid: Editorial Verbum.

Echevarría, A. (2013). La noria. La Habana: Ediciones Unión.

Gallardo Saborido, E. (2009). El Martillo y El Espejo: Directrices de La Política Cultural Cubana, 1959-1976. Madrid: Consejo Superior de Investigaciones Científicas.

Mellado, L. A. (2007). Representaciones del campo intelectual y literario en Las Iniciales de la tierra y Las palabras perdidas de Jesús Díaz. Alpha (Osorno), 24, pp. 95-109. doi: 10.4067/ So718-22012007000100007

Menton, S. (1975). Prose Fiction of the Cuban Revolution. Austin: University of Texas Press.
Navarro, D. eds. (2008). "Para una cronología". La política cultural del período revolucionario: memoria y reflexión. La Habana: Centro Teórico-Cultural Criterios.

Reed, R. (1991). The Cultural Revolution in Cuba. Latin American Round Table.

Rojas, R. (2009). El estante vacío: Literatura y Política en Cuba. Barcelona: Editorial Anagrama, 2009.

Simal, M. (2017). La noria de Ahmel Echevarría Peré o La máquina contra el olvido. Revista Letral, 18, pp. 56-75.

\section{Notas}

1 Emilio J. Gallardo (2009) hace un repaso general de los tres episodios que conformaron la polémica del "caso Padilla". El episodio de censura más conocido fue la presentación de su poemario Fuera del juego, obra interpretada como un enaltecimiento del sentimiento contrarrevolucionario. Padilla fue encarcelado y tuvo que hacer una alegación en público en la que tuvo que retractarse y "confesar" sus faltas. Para información más detallada sobre el caso: Roger Reed (1991). Por su parte, Arturo Arango expone que "los acontecimientos derivados de los dos casos Padilla y el Primer Congreso Nacional de Educación y Cultura provocaron daños esenciales a la cultura y a la Revolución cubanas. El más visible y doloroso de ellos fue la marginación, el castigo a un nutrido grupo de intelectuales de las más diversas manifestaciones, formas de expresión y tendencias artísticas" (Arango, 2008, pp. 116-117).

2 Antón Arrufat fue censurado por su obra Los siete contra Tebas tras recibir el Premio José Antonio Ramos de la UNEAC de 1968, al ser interpretado como un texto con "desviaciones ideológicas".

3 Reinaldo Arenas se exilió en Nueva York pero mientras vivió en la isla fue acosado y jamás se lo reconoció como escritor. Su escritura en la actualidad se analiza desde la perspectiva del trauma, entre otras.

4 P.M. (1961) es un cortometraje realizado por Alberto 'Sabá' Cabrera y Orlando Jiménez. Cabrera Infante lo describe como "una suerte de documental político, sin aparente línea argumental, que recoge las maneras de divertirse de un grupo de habaneros un día de fines de 1960 [...]. En la película se ven cubanos bailando, bebiendo y, en un 
momento de la peregrinación por bares y cabarets de "mala muerte", una pelea. Comienza temprano en la noche en Prado y termina en la madrugada al otro lado de la bahía, con el barquito regresando a La Habana de Regla” (Gallardo, 2009, p. 66). La Comisión que censuró el corto expuso que el motivo fue que "ofrecía una pintura parcial de la vida nocturna habanera que, lejos de dar al espectador una correcta visión de la existencia del pueblo cubano en esta etapa revolucionaria, la empobrecía desfiguraba y desvirtuaba” (Luis, (2003:224) en Lunes de Revolución, Verbum, como se citó en Gallardo, 2009, p. 67).

5 'Parametrado', persona separada de su puesto en un grupo teatral u otro medio cultural público por no cumplir con supuestos "parámetros" de comportamiento revolucionario establecidos en materia de orientación sexual, creencias, vestimenta, etc. (Navarro, 2008, p.7). 